\title{
Realidad de la Revista Chilena de Radiología 2010-2014
}

\author{
Dr. Marcelo Gálvez $\mathbf{M}^{(1)}$, Patricio Gana $\mathbf{G}^{(2)}$, Paulina Gana $\mathbf{P}^{(2)}$, Sonia Rivas $\boldsymbol{H}^{(3)}$.
}

1. Editor Científico de Revista Chilena de Radiología. Médico Radiólogo Clínica Las Condes. Santiago - Chile.

2. Editorial Publimpacto. Santiago - Chile.

3. Periodista, Sociedad Chilena de Radiología. Santiago - Chile.

\section{Chilean Journal of Radiology Facts 2010-2014}

\begin{abstract}
The purpose of this article is an editorial review of the Chilean Journal of Radiology in the period 2010 to 2014, for which the publication rates, rejections, source/origin of articles and types of work and research were analyzed. During this period 162 papers were published, of which 138 corresponded to original articles (30\%), revision (25\%) or clinical cases (30\%), while the rejection rate was $25 \%$. It's necessary to consider that the rejection or acceptance does not always correspond to the value of the information in the manuscript, as there are many and various factors that determine the decision to accept or reject a work. It was also observed that most of the topics refer to gastrointestinal pathology, showing that there is a deficit of studies about radiological techniques and interventional radiology. As to the sources, it was found that $23 \%$ come from abroad and $77 \%$ are national articles. Within the latter, $92 \%$ are from Santiago and only $8 \%$ are from regions. There were 1,875 citations given during the period, which corresponded mainly to North American magazines. The citations received in the period were 138, published in 60 journals, mainly from Chile (43\%) and Cuba (24\%). The impact factor calculated by Scientific Electronic Library On Line (SciELO) in 2014 was 0,083, with four citations received during the last year from 48 articles published between 2012 and 2013. It was also found that during the period under review the most widely read magazine article, which is index-linked in SciELO since 2002, has over 220,000 viewings.

At present, the Chilean Journal of Radiology is the sixth most downloaded scientific publication, exceeding 4 million requests for articles, through the ScIELO electronic library, which thus makes it the best presentation card for the Chilean Radiology Society. It must be noted that this publication is also index-linked in the Revista Virtual del Colegio Interamericano de Radiología CIR and in Literatura Latinoamericana y del Caribe en Ciencias de la Salud (LILACS).
\end{abstract}

Keywords: Bibliometric, Biomedical Publications, Scientific magazines, SciELO.

Resumen: El presente artículo tiene como finalidad la revisión editorial de la Revista Chilena de Radiología en el período 2010 al 2014. Durante el período se publicaron 162 artículos, de los cuales 138 fueron artículos originales, artículos de revisión o presentación de casos clínicos. Los artículos originales correspondieron a 30\% de las publicaciones, los no originales un $70 \%$. De estos últimos, $30 \%$ fueron casos clínicos y $25 \%$, artículos de revisión. Se rechazó el $25 \%$ de los artículos recibidos. El área de la Radiología con mayor representación fue la patología gastrointenstinal existiendo un déficit relativo de trabajos en áreas relativas a técnicas radiológicas y radiología intervencional. Los artículos nacionales representaron $77 \%$ y los internacionales el $23 \%$. Dentro de los artículos nacionales, el 92\% de los artículos fueron enviados de centros en Santiago y el $8 \%$ de regiones. Las citas concedidas en el período fueron 1875 y correspondieron principalmente a revistas norteamericanas. Las citas recibidas en el período fueron 138, publicadas en 60 revistas, provenientes mayoritariamente de Chile (43\%) y Cuba (24\%). La revista se encuentra indizada en Scielo desde el año 2002, en la Revista Virtual del Colegio Interamericano de Radiología CIR y en Literatura Latinoamericana y del Caribe en Ciencias de la Salud (LILACS). El factor de Impacto calculado por SciELO en el año 2014 fue de 0,083, con 4 citas recibidas el año 2014 de 48 artículos publicados en los años 2012 y 2013. El artículo más visitado en el período tiene más de 220.000 visitas. La Revista Chilena de Radiología es la sexta revista más descargada en el periodo, con más de 4 millones de peticiones de artículos. A través del portal ScieELO, la revista se ha convertido en una importante ventana al trabajo científico de la radiología chilena apoyado por su sociedad científica.

Palabras clave: Biobliometría, Publicaciones biomédicas, Revistas científicas, SciELO. 
Gálvez M, Gana PE, Gana PB, Rivas S. Realidad de la Revista Chilena de Radiología 2010-2014. Rev Chil Radiol 2015; 21(1): 26-33.

Correspondencia: Marcelo Gálvez M. / mgalvezm@gmail.com

Trabajo recibido el 06 de enero de 2015. Aceptado para publicación el 31 de marzo de 2015.

\section{Introducción}

El presente artículo tiene como finalidad la revisión editorial de la Revista Chilena de radiología en el período 2010 al 2014.

La misión de la Sociedad Chilena de Radiología es promover el desarrollo de la Radiología y ser un órgano de fomento y difusión científica, a través de la realización de actividades, entre las cuales la revista juega un rol importante que subraya y difunde los avances tecnológicos, la experiencia y la investigación clínica ${ }^{(1,2)}$. Si compartimos la opinión de Leonardo da Vinci, en el sentido de que "La ciencia más útil es aquella cuyos frutos se transmiten mejor", publicar debiera ser un trabajo inherente a la actividad científica.

Las publicaciones biomédicas constituyen un apoyo esencial para la actualización de los conocimientos y para ayudar en la toma de decisiones diaria a la que nos enfrenta la práctica médica.

\section{Artículos}

\section{Artículos publicados, aceptados y rechazados}

Durante los años 2010 al 2014 se publicaron 162 artículos en 20 números, con aproximadamente ocho artículos por número.

El trabajo editorial implica la circulación de una cantidad considerable de artículos en diferentes fases de aceptación, antes de alimentar un número en forma definitiva. Los artículos recibidos son distribuidos entre los revisores expertos, los que aportan su experiencia para asegurar la utilidad, novedad y calidad del material seleccionado. Los trabajos suelen ser devueltos a los autores para revisión y cambios cuando procede, sugerencias que pueden o no ser aceptadas por los autores. El material revisado ingresa a diagramación, proceso que más allá de distribuir proporcionalmente los espacios del texto entrega un sello visual que es único para cada revista.

En cada uno de estas etapas los trabajos pueden ser descartados. Durante el período 2011 al 2014 se recibieron 139 artículos de los cuales fueron publicados 109. Treinta y cinco artículos fueron rechazados, lo que traduce una tasa de rechazo para el periodo de $25,2 \%$ (Gráfico 1)

La tasa general de rechazo de una revista se relaciona con el interés en publicar en dicha revista. Publicaciones como Science, Nature o New England Journal of Medicine, llegan hasta $95 \%$ de rechazo, hecho que coincide con el gran prestigio de estas revistas y con el enorme interés de publicar en ellas ${ }^{(3)}$.

\section{Artículos por tipo (original, revisión, casos, editorial y otros)}

En general, los trabajos científicos se clasifican

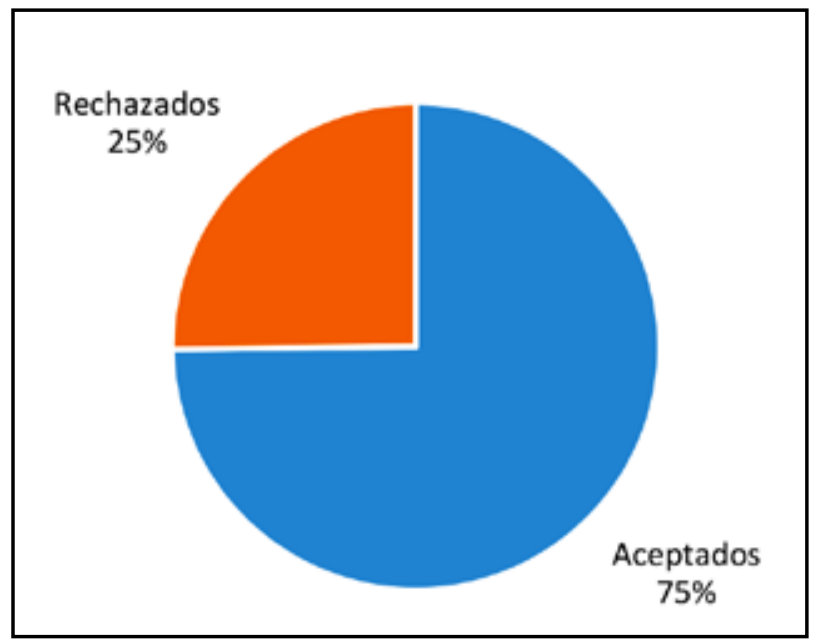

Gráfico 1. Artículos recibidos 2011-2014.

en artículos Originales y No Originales. Los artículos originales corresponden al reporte escrito de la investigación llevada a cabo por una persona o un grupo de investigadores, y que es realizado por el o los responsables del estudio. Se consideran fuentes primarias de información. Los artículos No Originales, consideran principalmente revisión de trabajos publicados previamente y la presentación de casos clínicos. En general, se consideran como citables los artículos originales y los artículos de revisión.

Los artículos originales, los artículos de revisión y los casos clínicos publicados durante el período correspondieron a 138 artículos. De ellos SciELO sólo reconoció como citables 125 artículos (Tabla I).

\begin{tabular}{|c|c|c|c|c|c|c|}
\hline Tipo/Año & 2010 & 2011 & 2012 & 2013 & 2014 & Total \\
\hline Originales & 8 & 14 & 9 & 10 & 8 & 49 \\
\hline Revisiones & 13 & 6 & 5 & 9 & 7 & 40 \\
\hline Casos & 8 & 9 & 14 & 7 & 11 & 49 \\
\hline Subtotal & 29 & 29 & 28 & 26 & 26 & 138 \\
\hline Editoriales & 4 & 4 & 4 & 4 & 4 & 20 \\
\hline Otros & 1 & 0 & 0 & 0 & 3 & 4 \\
\hline Total & 34 & 33 & 32 & 30 & 33 & 162 \\
\hline
\end{tabular}

Respecto de los artículos originales publicados por nuestra revista, el Congreso Chileno de Radiología es su principal fuente de origen. Sin embargo, el entusiasmo por comunicar resultados de trabajos originales durante 
el Congreso no tiene relación directa con el número de trabajos efectivamente enviados para publicación, tal como lo comentó en una de las editoriales el Dr. José Domingo Arce, a cargo del trabajo editorial de la revista durante cinco años ${ }^{(4)}$. Los artículos originales publicados durante el período alcanzan el 30\%. Revistas indizadas en Medline tienen, por su parte, porcentajes mayores al $40 \%$.

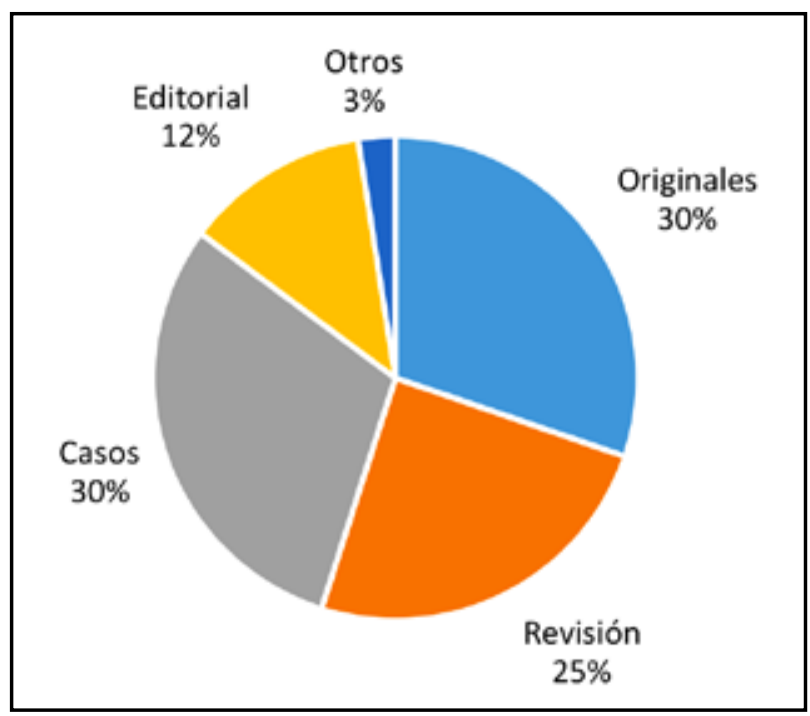

Gráfico 2. Artículos publicados según Tipo 2010-2014.

El Dr. Mauricio Canals en la publicación donde analizó la investigación en Radiología estudió el impacto nacional e internacional de 100 radiólogos primeros autores de los artículos, concluye que nuestra actividad científica reportada consiste principalmente en trabajos de revisión de la literatura y en reporte de $\operatorname{casos}^{(5)}$.

La presentación de casos clínicos es una sección que ha sido abandonada por muchas de las principales revistas o que representa un porcentaje pequeño, en algunas menos del $10 \%$, de su material editorial ${ }^{(6)}$. En nuestra revista correspondió al $30 \%$ durante el período de estudio. Sin embargo, a pesar de su menor peso editorial, este tipo de publicaciones han servido como canal de difusión de los esfuerzos académicos, de varios grupos de radiólogos, tal como lo ha destacado el Dr. Claudio Silva ${ }^{(7)}$.

La sección de Signos Radiológicos comenzó su publicación durante el año 2009, durante el período de trabajo editorial de la Dra. Karla Moënne. Estos reportes, corresponden a comunicaciones breves de los signos que utilizamos en el trabajo radiológico diario $^{(8)}$. En el período 2010 al 2014 se publicaron 13 artículos con estas características.

\section{Artículos clasificados por áreas}

Durante el período se estudió la representación de las diferentes subespecialidades de la Radiología en el material publicado por la revista, como se resume en el Gráfico 3. La representación deja de manifiesto cómo la patología gastrointestinal concentra el mayor número de artículos publicados, correspondiendo aproximadamente al $25 \%$ de los artículos publicados (34/138).

Las áreas con menor representación correspondieron a Técnica Radiológica y Radiología Intervencional. Estas áreas tienen la trascendencia del manejo de las técnicas y sus innovaciones, por otro lado el mandato de una subespecialidad requerida, necesaria y creciente como es la radiología intervencional. Sin duda existe entonces un déficit de artículos en estas dos áreas que debiera suplirse durante los próximos años con la estabilización de las nuevas técnicas y con un mayor número de especialistas y procedimientos intervencionales.

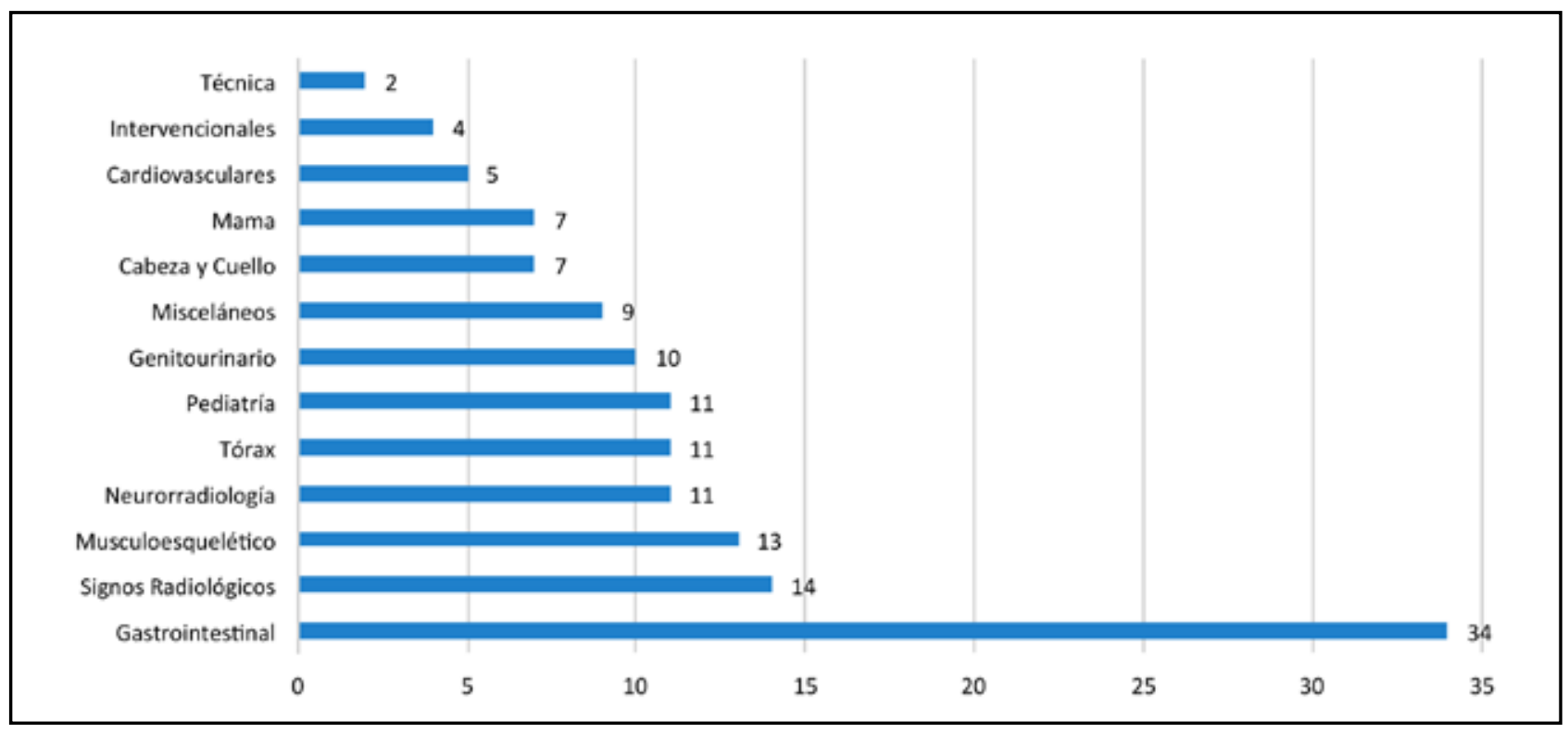

Gráfico 3. Artículos publicados según área. 


\section{Artículos clasificados por geografía}

Los artículos publicados durante el período se clasificaron según la ubicación de los centros a los que pertenecían los autores. La mayoría de los trabajos publicados se originaron en centros nacionales, representado los artículos extranjeros casi un cuarto de los artículos del período (Gráfico 4).

De los artículos nacionales, el aporte de autores de regiones es menor al $10 \%$ en los artículos publicados en la revista, lo que contrasta con el $47 \%$ que ellos representan del total de radiólogos del país ${ }^{(9)}$ (Gráfico 5).

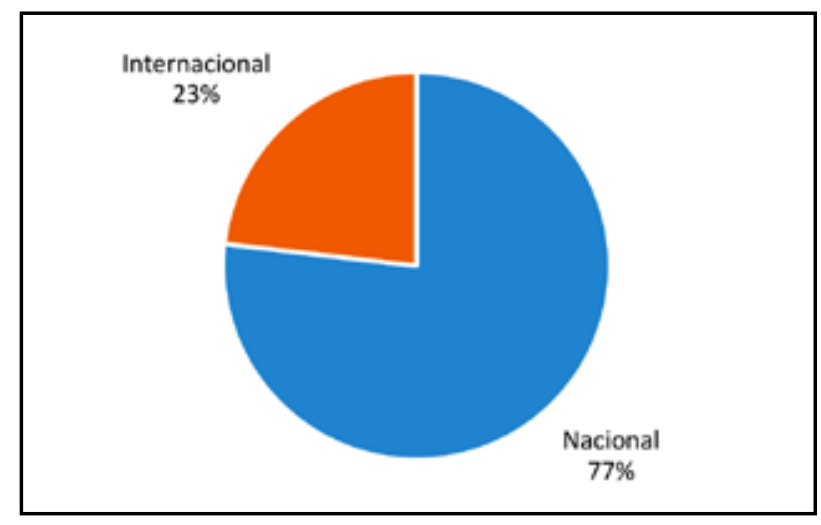

Gráfico 4. Artículos publicados según países.

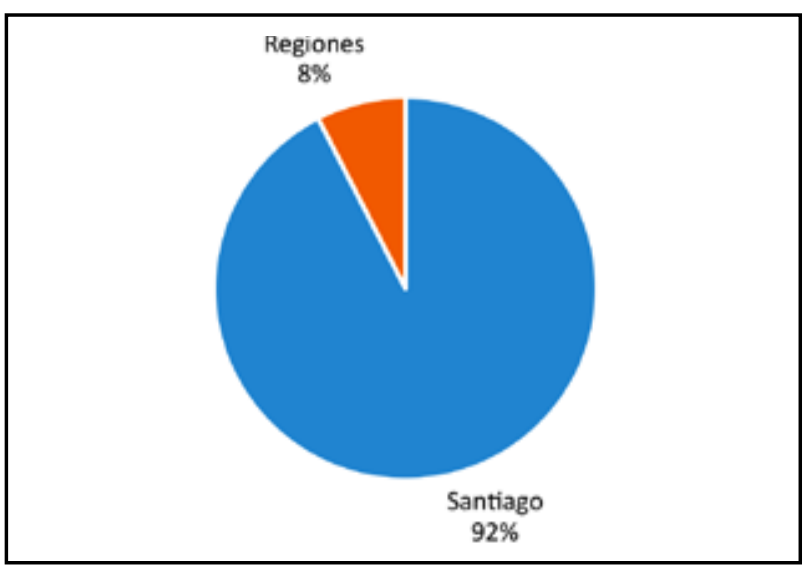

Gráfico 5. Artículos nacionales publicados 2010-2014.

\section{Autores más publicados}

No existe una guía formal sobre cómo deben ordenarse los autores de una publicación científica. Así por ejemplo, se considera que el lugar del primer autor está reservado para el investigador que más trabajó en el estudio y en su reporte, que conoce y es responsable de todos los detalles del manuscrito; en forma consistente, será también el autor más citado. Sin embargo, de acuerdo a preferencias locales, existen centros, donde los líderes de grupos académicos o de centros de investigación se colocan rutinariamente al final del listado.
Los primeros autores de los 138 artículos publicados durante el período fueron 111 profesionales, de los cuales 13 publicaron más de un artículo, según consta en la Tabla II. Este grupo de radiólogos representó aproximadamente el $29 \%$ de la producción total de la revista para el mismo período.

Tabla II. Número de publicaciones por autor 20102014.

\begin{tabular}{|ccc|}
\hline № de & № de & Total № \\
\hline artículos /autor & autores & artículos \\
\hline & & \\
\hline 5 & 2 & 10 \\
\hline 4 & 2 & 8 \\
\hline 3 & 4. & 12 \\
\hline 2 & 5 & 10 \\
\hline 1 & 98 & 98 \\
\hline Total & 111 & 138 \\
\hline
\end{tabular}

\section{Citas}

El proceso de poner citas en el cuerpo del artículo que se está escribiendo o el hecho de ser citado por publicaciones de otros autores representa uno de los momentos más trascendentes del reporte, ya que ambos involucran avanzar en la ciencia de la mano de otros investigadores. Citar significa reconocer a nuestras fuentes en la tarea de dar crédito a nuestras observaciones y hallazgos, aprendiendo sobre el contexto en el cual nuestro reporte se abre espacio. Ser citado por otro autor, representa la oportunidad de participar en el contexto de otro grupo de trabajo, de sustentar sus afirmaciones o de abrir alternativas para que los lectores profundicen contenidos.

\section{Citas concedidas}

En el período 2010-2014 SciELO reconoce 125 artículos como citables, con un total de 1.875 referencias concedidas. Las citas por artículo se estimaron en 15 en promedio para el período de evaluación. Dentro de las referencias se destaca la preferencia de revistas norteamericanas como Radiology, AJR y Radiographics (Tabla III).

\section{Citas recibidas}

Uno de los factores más importantes en la evaluación de calidad de una revista es cuánto citan a sus autores. Desde el año 2002 en que la revista hace su ingreso en el portal SciELO se reconocen 60 artículos citados.

Si SciELO reconoce 125 artículos como citables, tenemos como promedio algo más de una cita por artículo en 5 años. Las citas provienen de las siguientes revistas, indicadas en la Tabla IV. 
Tabla III. Citas concedidas Revista Chilena Radiología 2010-2014.

\begin{tabular}{|rlc|}
\hline № & Revista & Citas \\
\hline & & \\
\hline 1 & Radiology & 136 \\
\hline 2 & American Journal of Röentgenology & 126 \\
\hline 3 & Radiographics & 122 \\
\hline 4 & Journal of Magnetic Resonance Imaging & 29 \\
\hline 5 & Pediatric Radiology & 22 \\
\hline 6 & Revista Chilena de Radiología & 22 \\
\hline 7 & The New England Journal of Medicine & 20 \\
\hline 8 & Revista Médica de Chile & 19 \\
\hline 9 & Circulation & 17 \\
\hline 10 & European Radiology & 16 \\
\hline
\end{tabular}

Tabla IV. Citas recibidas en Revista Chilena de Radiología periodo 2010-2014.

\begin{tabular}{|llc|}
\hline № & Revista & n \\
\hline & & \\
\hline 1 & Revista Chilena de Radiología & 22 \\
\hline 2 & Revista Chilena de Cirugía & 9 \\
\hline 3 & Revista Médica de Chile & 7 \\
\hline 4 & Medisan & 6 \\
\hline 5 & Revista Chilena de Infectología & 6 \\
\hline 6 & Revista Archivo Médico de Camaguey & 5 \\
\hline 7 & Revista Argentina de Radiología & 5 \\
\hline 8 & Revista de Ciencias Médicas de Pinar del Río & 5 \\
\hline 9 & Gen & 3 \\
\hline 10 & Revista Chilena de Enfermedades Respiratorias & 3 \\
\hline 11 & Revista Chilena de Pediatría & 3 \\
\hline 12 & Revista Cubana de Medicina Militar & 3 \\
\hline
\end{tabular}

\section{Citas recibidas por país}

El origen geográfico de las citas recibidas da cuenta de otro de los factores importantes de una revista y su influencia en la región. Los países que se destacan en términos de cantidad de revistas científicas son: Brasil, México, Colombia, Chile, Argentina y Venezuela ${ }^{(10)}$. Las citas recibidas de nuestros artículos provienen principalmente de Chile, Cuba, Brasil, Colombia y España (Gráfico 6).

\section{Factor de impacto SciELO}

La calidad e impacto de una investigación no son sinónimos. La primera se refiere al contenido científico, su originalidad, metodología, importancia y relevancia de los resultados, mientras que el impacto es la influencia que la publicación puede tener sobre la investigación general en un momento determinado

El factor de impacto (FI) de una revista es un instrumento que sirve para comparar y evaluar la

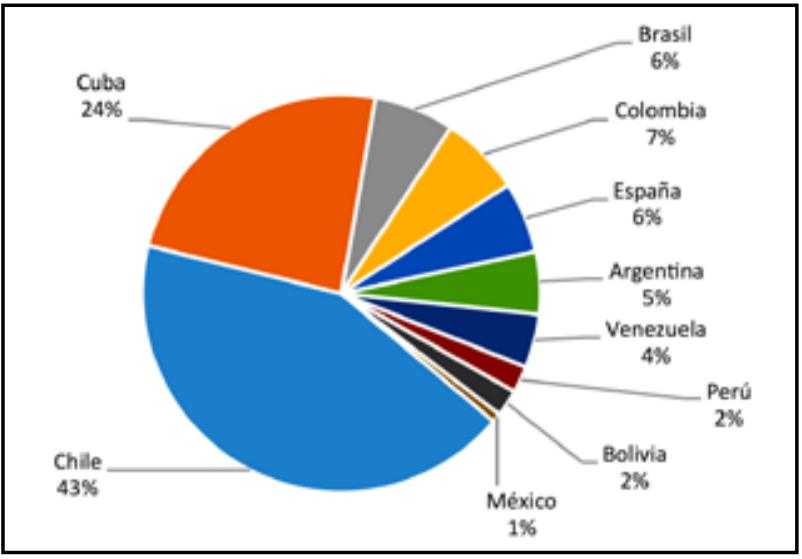

Gráfico 6. Citas recibidas revista Chilena Radiología 20102014 según país.

importancia relativa de una revista dentro de un mismo campo científico.

\section{Ejemplo:}

Factor de Impacto 2014= A/B

$\mathrm{A}=\mathrm{N}^{\circ}$ de veces en que los artículos publicados en la revista en el período 2012-2013 han sido citados por las publicaciones a las que se les da seguimiento durante el año 2014

$\mathrm{B}=$ Total de artículos publicados en la revista en el período 2012-2013.

El factor de impacto de algunas revistas médicas chilenas y su relación con las citas durante el período 2012-2013 se ha resumido en la Tabla V.

Tabla V. Factor de Impacto 2014 de Revistas Chilenas. Citas recibidas el año 2014 de artículos publicados en los años 2012 y 2013.

\begin{tabular}{|lccc|}
\hline Revista & $\begin{array}{c}\text { Artículos } \\
\text { publicados } \\
\text { 2012-2013 }\end{array}$ & $\begin{array}{c}\text { Citas } \\
\text { recibidas } \\
\mathbf{2 0 1 4}\end{array}$ & $\begin{array}{c}\text { Factor } \\
\text { Impacto } \\
\mathbf{2 0 1 4}\end{array}$ \\
\hline Rev Chil Nutr & 100 & 35 & 0,351 \\
\hline Rev Chil Infectol & 189 & 51 & 0,270 \\
\hline Rev Med Chil & 415 & 108 & 0,260 \\
\hline Rev Chil Enferm Respir & 50 & 13 & 0,210 \\
\hline Int J Morphol & 491 & 103 & 0,210 \\
\hline Rev Chil Pediatr & 115 & 23 & 0200 \\
\hline Rev Chil Cir & 157 & 31 & 0,197 \\
\hline Rev Chil Obstet Ginecol & 144 & 20 & 0,139 \\
\hline Rev Chil Neuro Psiquiatr & 53 & 6 & 0,113 \\
\hline Rev Chil Radiol & 48 & 4 & 0,083 \\
\hline
\end{tabular}

\section{Indización}

La indización de las revistas biomédicas tiene exigencias específicas asociadas a estándares internacionales que garantizan calidad y que permiten 
su reconocimiento internacional. Existen diferentes niveles de indización que van desde exigencias menores hasta niveles muy exigentes. Nuestra revista se encuentra indizada en LILACS, SciELO y aporta artículos la Revista Virtual de Radiología. Los siguientes niveles de indización corresponden a MEDLINE y luego ISI cuyos estándares son más exigentes y que sólo algunas revistas chilenas los alcanzan actualmente.

\section{a. LILACS}

Nacido hace 29 años, es el índice de Literatura Latinoamericana y del Caribe en ciencias de la Salud más importante de la región. Nuestra revista se encuentra indexada desde el año 1998 y es el nivel básico de indización en nuestra región.

\section{b. SCIELO}

El proyecto de SciELO es una iniciativa de FAPESP (Fundación de Apoyo a la Investigación del Estado de Sao Paulo) y de BIREME (Biblioteca Regional de Medicina o su nombre actual Centro Latinoamericano y del Caribe de información de Ciencias de la Salud). Contempla una metodología común para la preparación, almacenamiento, diseminación y evaluación de las bibliotecas científicas en formato electrónico.

La representación científica de Chile en esta iniciativa nacida en Brasil está a cargo de la Comisión Nacional de Investigación Científica y Tecnológica (CONICYT). Su incorporación, logro alcanzado gracias a los esfuerzo del Dr. Gonzalo Delgado(11) ha sido uno de los hechos más importante en la difusión de la revista, permitiendo su existencia en línea y aumentado significativamente su visibilidad.

\section{c. Revista Virtual del Colegio Interamericano de Radiología}

La revista virtual de Radiología ha sido una iniciativa realizada por el Colegio Interamericano de Radiología en el año 2010 en la cual participó la Dra. Karla Moënne, como Editora Científica de la Revista. Durante el período 2010-2014 se publicaron 19 números, con 38 artículos publicados en la Revista Chilena de Radiología que han sido traducidos al inglés y que se encuentran en línea en el portal http://www.webcir.org/revistavirtual/(12).

\section{d. Medline / PubMed}

MEDLINE ${ }^{\circledR}$ contiene citas y resúmenes de literatura biomédica de todo el mundo. PubMed ${ }^{\circledR}$ proporciona acceso gratuito a MEDLINE y enlaces a las respectivas revistas o a artículos completos cuando estos se encuentran disponibles. Muestra el número de artículos publicados en revistas nacionales e internacionales de revistas indexadas en PubMed durante el año 2005 y su relación con el número de habitantes. En relación al número total de artículos indexados en MEDLINE en el año 2005, la región contribuye sólo con el $2,16 \%$ del total de trabajos ${ }^{(13)}$.

\section{e. ISI (Journal Citation Report).}

El Institute for Scientific Information, fundado en 1960, mantiene una base de datos de citas que cubre miles de revistas. Ofrece servicios de bibliografía y está particularmente especializado en el análisis de citación. ISI publica anualmente el Journal Citation Report que lista el factor de impacto de cada una de las revistas que controla.

La Tabla VI resume el estado respecto de indización de las principales revistas médicas chilenas.

\begin{tabular}{|lll|}
\hline \multicolumn{3}{|l|}{ Tabla VI. Indización de las Revista Médicas Chilenas. } \\
\hline Rev Med Chile & SciELO & Medline $\quad$ ISI \\
\hline Rev Chil Pediatr & SciELO & Medline \\
\hline Rev Chil Infectol & SciELO & Medline \\
\hline Rev Chil Obstet Ginecol & SciELO & \\
\hline Rev Chil Nutr & SciELO & \\
\hline Rev Chil Radiol & SciELO & \\
\hline Int J Morphol & SciELO & \\
\hline Rev Chil Cir & SciELO \\
\hline Rev Chil Neuro Psiquiatr & SciELO \\
\hline Rev Chil Enferm Respir & SciELO \\
\hline
\end{tabular}

\section{VISITAS}

Sin duda la revista ha aumentado significativamente la visibilidad de los trabajos publicados, más allá del número de radiólogos del país y de los asistentes de todas las actividades realizadas por la Sociedad de Radiología.

El número de visitas ha aumentado significativamente año a año, con más de 5,5 millones de visitas entre el 2010-2014, como lo demuestra el Gráfico 7. En el período 2002-2003, con sólo seis números, el número de visitas era inferior a mil(14). Desde el año 2010, se supera y mantiene por sobre 1.000 el número de visitas.

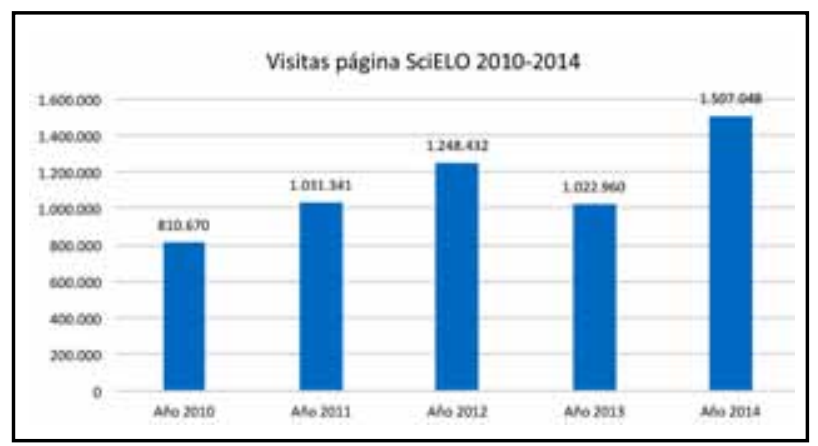

Gráfico 7. Número de visitas a la página web de la Revista Chilena de Radiología en SciELO durante los años 2010-2014. 
En la Tabla VII aparecen los artículos más visitados durante el período de estudio, los que en conjunto abarcan el $20 \%$ de las visitas a la revista.

La Revista Chilena de Radiología es la sexta revista con más descargas desde SciELO, con más de 4 millones de descargas en el período (Gráfico 8).

\section{Investigación en latinoamérica}

Sudamérica da cuenta del 5-6\% de la población y PIB del planeta. Sin embargo, aporta el $4 \%$ de los artículos de investigación a nivel mundial. Según un artículo de Nature ${ }^{(25)}$ Sudamérica es el continente oculto debido a que su poder de investigación puede ser subestimado. Esto sucedería debido a que sus investigadores publican en revistas que no están indexados en las principales bases de datos de citas. Elevar el estándar de la Revista Chilena de Radiología al de una publicación científica de clase mundial requiere un trabajo que incluye obviamente el desempeño prolijo del equipo editorial, pero que por sobre todo, debe nutrirse de la actividad científica creciente y fecunda de todos los centros nacionales.

\section{Comentarios}

La Revista Chilena de Radiología cuenta con más de 20 años de historia y paulatinamente ha mejorado en calidad, encontrándose actualmente indizada en LILACS, SciELO y aporta artículos a la Revista Virtual del Colegio Interamericano de Radiología (CIR).

Los artículos originales y artículos de revisión corresponden al $55 \%$ de nuestros trabajos. Dentro de las áreas publicadas tenemos un número deficiente de trabajos en técnicas radiológicas y radiología intervencional.

El rechazo de artículos alcanza un 25\%.

Desde el punto de vista del origen un $23 \%$ de nuestros artículos son de autores extranjeros, sin embargo, los autores nacionales se concentran en Santiago (92\%).

Nuestra Revista ha recibido más de 5,5 millones de visitas y más de 4 millones de descargas en el período 2010-2014, siendo la sexta revista en número de descargas del país.

Tabla VII. Artículos más visitados entre los años 2010-2014.

\begin{tabular}{|c|c|c|c|c|}
\hline № & Título & Autor & Año & Visitas \\
\hline 1 & $\begin{array}{l}\text { Elementos de osteosintesis de uso habitual en fracturas } \\
\text { del esqueleto apendicular: Evaluación radiológica }\end{array}$ & García M, César(15) & 2005 & 224.290 \\
\hline 2 & Errores neurorradiológicos frecuentes en TC y RM & Orellana P Patricia & 2003 & 156.239 \\
\hline 3 & Lesiones musculares deportivas: Diagnóstico por imágenes & Muñoz Ch. Sara & 2002 & 143.288 \\
\hline 4 & $\begin{array}{l}\text { Características de las hemorragias intracraneanas } \\
\text { espontáneas en TC y RM }\end{array}$ & Galvéz M, Marcelo & 2007 & 103.777 \\
\hline 5 & $\begin{array}{l}\text { Lesiones deportivas osteocartilaginosas en el niño y } \\
\text { adoslescente }\end{array}$ & Pose L, Georgette & 2005 & 99.357 \\
\hline 6 & $\begin{array}{l}\text { Estudios de imágenes de articulación patelofemoral. } \\
\text { ¿En que estamos? }\end{array}$ & Miranda V, Edith & 2010 & 78.147 \\
\hline 7 & Inestabilidad glenohumeral: Lo que el radiológo debe saber & Zamorano C, Carolina & 2009 & 74.113 \\
\hline 8 & $\begin{array}{l}\text { Gases de abdomen su utilidad diagnóstica: } \\
\text { Gases endoluminales (Segunda parte) }\end{array}$ & Cortés A, Claudio & 2002 & 74.002 \\
\hline 9 & Actualización de la evaluación radiológica de la escoliosis & Díaz J, Jorge & 2009 & 68.520 \\
\hline 10 & $\begin{array}{l}\text { Signos de rotura del ligamento cruzado anterior en } \\
\text { radiografía simple }\end{array}$ & Rabat J, Carolina & 2008 & 54.686 \\
\hline
\end{tabular}

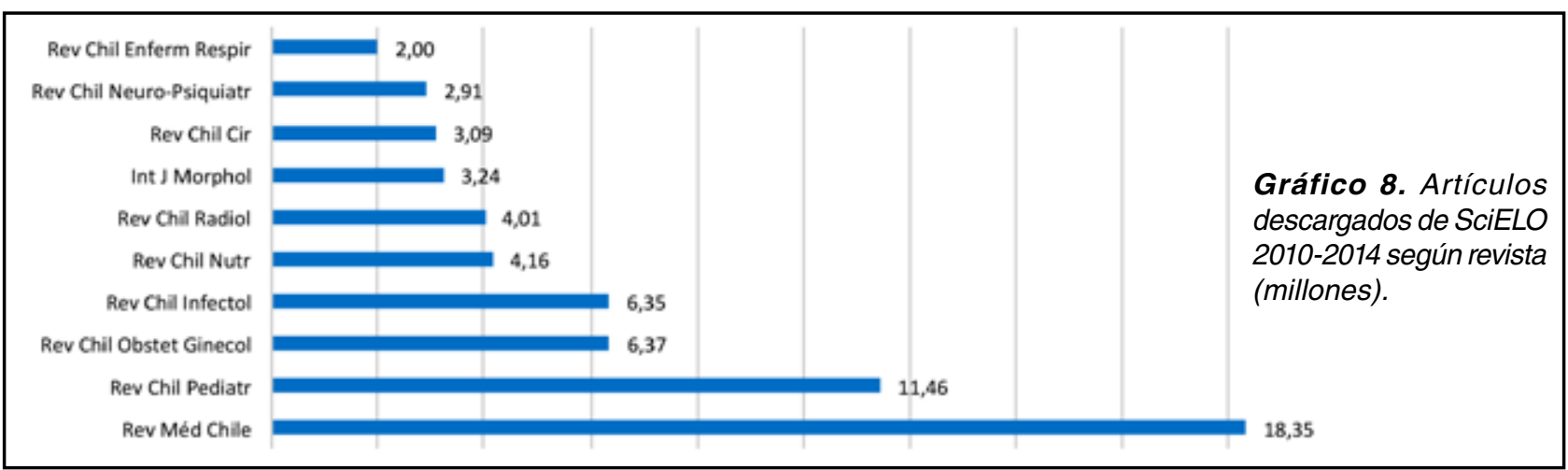




\section{Bibliografía}

1. Castro M, Gana P. Encuesta Radiológica. Rev Chil Radiol 2001; 7(1): 27-30.

2. Galvez M. Encuesta radiológica. Rev. Chil. Radiol 2007; 13(4): 218-220.

3. Young P, Trimarchi H. Carta al Editor. Rev Med Chile 2013; 141(7): 940-941

4. Arce J. Editorial. Rev Chil Radiol 2002; 8(1): 3.

5. Canals M. Ciencia e imaginología: Análisis cuantitativo de la investigación radiológica. Rev Chil Radiol 2008; 14(4): 221-226.

6. Harris P, Liebbe JL, Sotomayor J, Ugarte F, Cano F. Análisis comparativo y perfil de publicaciones en la Revista Chilena de Pediatría 2001-2006. Rev Chil Pediatr 2007; 78(3): 268-276.

7. Silva C. Reporte de casos - Relevancia Actual. Editorial. Rev Chil Radiol 2014; 20 (3): 89.

8. Moënne K. Nueva sección en la revista: Signos radiológicos. Rev Chil Radiol 2009; 15(2): 59.

9. Vergara ML, Osses E. Mapa de los radiólogos de Chile. Rev Chil Radiol 2007; 13(2): 70-74.

10. Miguel S. Revistas y producción científica de América Latina y el Caribe: su visibilidad en SciELO, RedALyC y SCOPUS. Revista Interamericana de Bibliotecología 2011; 34(2): 187-199.

11. Delgado G. Editorial. Rev Chil Radiol 2002; 8(4): 147.

12. Moënne K. Revista Virtual de Radiología-CIR. Rev Chil Radiol 2010; 16(1): 3

13. Gálvez M. Publicaciones biomédicas: Realidad de Chile y Latinoamérica. Rev Chil Radiol 2006; 12(3): 113-117, 171.
14. Arce J. Editorial. Rev Chil Radiol 2003; 9(4): 171.

15. García C, Ortega D. Elementos de osteosíntesis de uso habitual en fracturas del esqueleto apendicular: evaluación radiológica. Rev Chil Radiol 2005; 11(2): 58-70.

16. Orellana P. Errores Neurorradiológicos frecuentes en TC y RM. Rev Chil Radiol 2013; 9(2): 93-103.

17. Muñoz S. Lesiones musculares deportivas: diagnóstico por imágenes. Rev Chil Radiol 2002; 8(3); 127-132.

18. Gálvez M, Bravo E, Rodríguez P, Farías M, Cerda J. Características de las hemorragias intracraneana espontáneas en TC y RM. Rev Chil Radiol 2006; 12(4); 12-25.

19. Pose G. Lesiones deportivas osteocartilaginosas en el niño y adolescente. Rev Chil Radiol 2005: 11(2); 91-100.

20. Miranda E, Muñoz S, Paolinelli P, Astudillo C, Wainer M, Duboy J. Estudio de imágenes de articulación patelofemoral: ¿En qué estamos? Rev Chil Radiol 2010; 16(3); 101-115.

21. Zamorano C, Munoz S, Paolinelli P. Inestabilidad glenohumeral: lo que el radiologo debe saber. Rev Chil Radiol 2009; 15(3): 128-140.

22. Cortes C. Gases del abdomen su utilidad diagnóstica: gases endoluminales (segunda parte). Rev Chil Radiol 2002; 8(3); 107-118.

23. Diaz J, Schroter C, Schulz R. Actualizacion de la evaluación radiológica de la escoliosis. Rev Chil Radiol 2009; 15(3): 141-151.

24. Rabat C, Delgado G, Bosch E. Signos de rotura del ligamento cruzado anterior en radiografía simple. Rev Chil Radiol 2008; 14(1): 11-13.

25. Van Noorden R. South America by the numbers. Nature 2014; 510: 202-203.

\section{Tecnología superior para el diagnóstico e informe remoto}

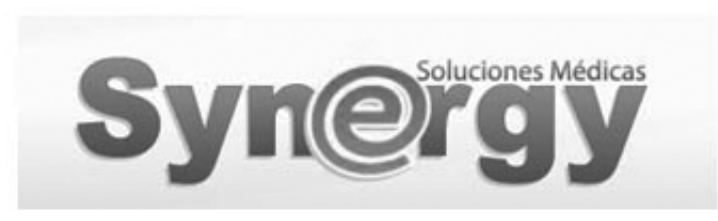

$>$ Las mejores soluciones integrales para las necesidades y requerimientos de profesionales médicos, que proveen servicios de diagnóstico e informe remoto a terceros.

> Configuramos la solución precisa para que optimice la productividad y confiabilidad del trabajo diagnóstico remoto.

> Precios verdaderamente convenientes y accesibles.
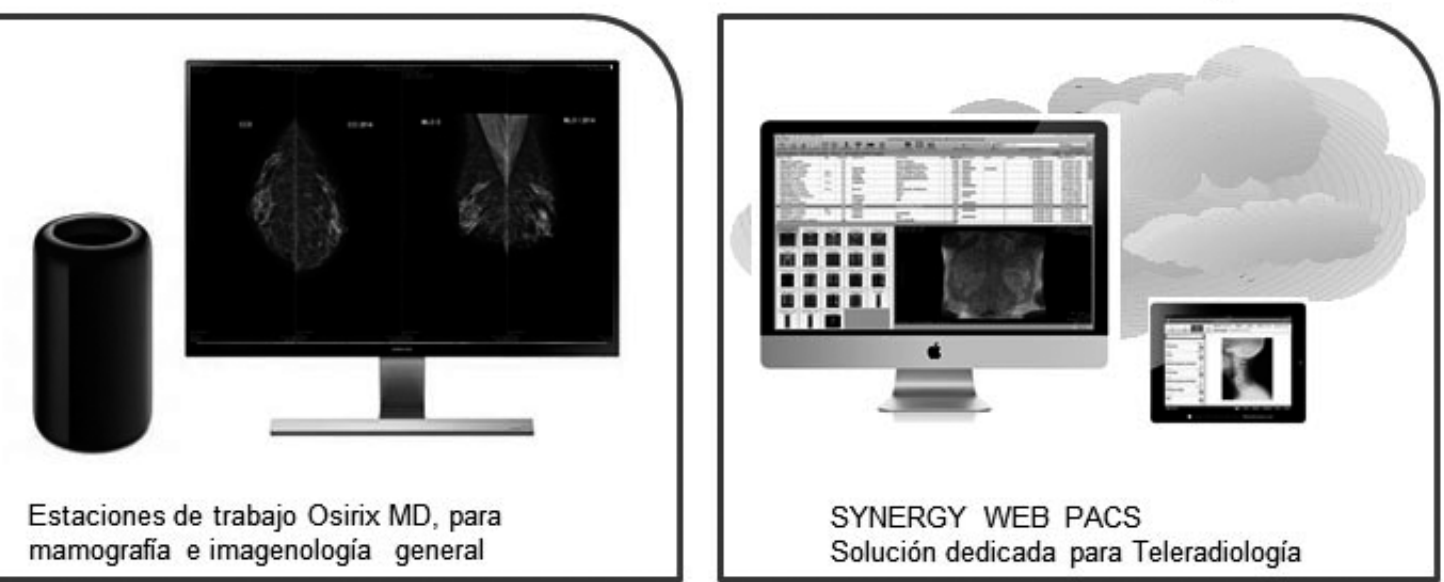

Estaciones de trabajo Osirix MD, para mamografia e imagenología general

Solución dedicada para Teleradiología

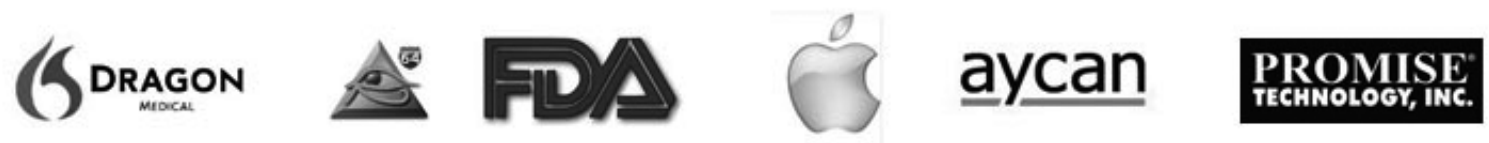

Synergy S.A. - Alférez Real 1154, Providencia- Santiago Tel: +562 28987400 - Fax: +56228987401-consultas@synergy.cl 